\title{
TV/Series
}

19 | 2021

Perfectionnisme et séries télévisées. Hommage à Stanley Cavell (1926-2018)

\section{Le quotidien des Girls et la morale de l'ordinaire}

\section{Elodie Valkauskas et Jean-Marc Leveratto}

\author{
(2) OpenEdition \\ Journals \\ Édition électronique \\ URL : https://journals.openedition.org/tvseries/5395 \\ DOI : 10.4000/tvseries.5395 \\ ISSN : 2266-0909 \\ Éditeur \\ GRIC - Groupe de recherche Identités et Cultures
}

Référence électronique

Elodie Valkauskas et Jean-Marc Leveratto, « Le quotidien des Girls et la morale de l'ordinaire », TV/ Series [En ligne], 19 | 2021, mis en ligne le 06 mai 2021, consulté le 14 mai 2021. URL : http:// journals.openedition.org/tvseries/5395; DOI : https://doi.org/10.4000/tvseries.5395

Ce document a été généré automatiquement le 14 mai 2021.

\section{(c)}

$T V /$ Series est mis à disposition selon les termes de la licence Creative Commons Attribution - Pas d'Utilisation Commerciale - Pas de Modification 4.0 International. 


\title{
Le quotidien des Girls et la morale de l'ordinaire
}

\author{
Elodie Valkauskas et Jean-Marc Leveratto
}

\section{Introduction : L'étude de la « réception » des séries télévisées : expertise profane et expertise sociologique}

1 L'institutionnalisation en France des «études de réception » a contribué à la routinisation d'une posture cognitive dommageable pour l'analyse sociologique de l'expérience artistique, la substantialisation conjointe du «public » et de «l'œuvre». Confirmant leur séparation objective, elle justifie leur observation per se, et le geste de réparation que constitue, d'un côté, l'enquête rendant compte des «usages sociaux » par un public déterminé d'un genre d'œuvres ${ }^{1}$ et, de l'autre, l'étude de l'« interactivité » qu'une œuvre déterminée d'art établit avec son public ${ }^{2}$. Il en résulte la neutralisation conjointe de l'attention du public au plaisir artistique et de la dimension éthique de la médiation artistique. Ceci explique le tournant anthropologique opéré par certains sociologues français, Antoine Hennion notamment, dès les années $1980^{3}$, pour sortir des limites de la sociologie des « pratiques culturelles » et son indifférence tout à la fois aux qualités techniques et aux émotions qui justifient pour les personnes l'engagement dans la consommation culturelle. Parler de tournant anthropologique s'impose. Il ne s'agit pas, en effet, simplement de prôner l'observation qualitative de la « réception » mais de remettre en cause une posture d'observation qui consiste à étudier les personnes indépendamment des objets qui alimentent la réception et leur procurent, selon les cas, plus ou moins de satisfaction. Ces objets auxquels les consommateurs réagissent, avec lesquels ils agissent et par le moyen desquels ils interagissent, importent particulièrement et demandent d'autant plus à être identifiés qu'ils « marchent " plus ou moins bien. La sociologie pragmatique, et son approche de l'art « en tant qu'expérience », interdit en ce sens de réduire l'art au seul projet de l'artiste ou au seul discours du public, et l'efficacité esthétique à la 
seule «magie» sociologique de l'harmonie préétablie entre objet et un sujet. Considérer l'acte de consommation sous l'angle de sa répétition volontaire dans la durée d'une vie ou d'un âge de la vie et en tant que moyen de familiarisation avec une technique, c'est appréhender le goût comme un "faire » (Hennion) qui génère une compétence et des instruments de mesure de la qualité de ce qu'on consomme, instruments de mesure dont le corps fait partie. Le terme d'expertise est plus adéquat, dans cette perspective, pour désigner la réception au sens typique (Shütz) que le terme de réception lui-même. Parler de réception, c'est parler d'une suite ou d'une série d'«expériences", au sens de situations vécues, qui sont l'occasion de "faire l'expérience ", au sens de ressentir la présence de certains objets, et d'" éprouver ", au sens de soumettre à une épreuve, leur qualité ${ }^{4}$. Tout cela renvoie à des sujets plus ou moins compétents techniquement, mais tous conscients des limites de leur expertise, et à des objets de plus ou moins grande qualité, qualité difficile à fixer du fait de leur caractère pléthorique, de la diversité des critères d'évaluation, et de leur dépendance des situations. Cette réception se laisse difficilement réduire à l'adhésion passionnée à un " champion », Godard contre Spielberg, La Joconde contre Le carré noir sur fond blanc, ou au confort paresseux de la consommation d'un genre indifférente à l'originalité technique et à l'élaboration idéologique de l'objet. Ni la création artistique (le chef d'œuvre), ni le contenu rituel (le genre de consommation) ne rendent compte de l'expérience de la consommation culturelle. L'objet consommé n'est pas seulement un acteur technique et un mobile psychologique. Il est, au cas par cas, pour tout consommateur un instrument de mesure et un enjeu - le plaisir que doit procurer son incorporation - de ce qui reste une transaction économique, au regard de la dépense de temps qu'exige sa consommation. L'acte de consommation est, de ce point de vue, une épreuve consciente par le spectateur de la singularité tant de l'action d'objets auxquels il rend son corps sensible que des réactions de son corps à la situation dans laquelle il est engagé.

2 De ce point de vue, on ne peut qu'adhérer à l'invitation de Louis Quéré, dès les années 1990, de «reconceptualiser le phénomène de la réception pour le conforter comme objet d'investigation des sciences sociales » en se rendant attentif à sa triple nature d' "activité située», d' "acte configurant», et d' "appropriation ${ }^{5}$ ». Elle s'inscrit, comme il le souligne lui-même, dans la continuité de l'École de Constance, de «l'esthétique de la réception»comprise comme un projet d'observation des « différentes manières dont le récepteur est affecté par ce qu'il lit, entend ou voit, y réagit et investit sa compréhension dans ses pratiques ${ }^{6} »$. Cette posture continue, cependant, à enfermer le risque d'une interprétation sociologique déterministe qui fait disparaitre la singularité de l'expérience du spectateur, alors même que le choix de l'observation ethnographique vise à rendre compte, et rend compte effectivement, de la diversité des «manières dont le récepteur est affecté et investit sa compréhension dans ses pratiques ». L'œuvre ou le public sélectionné pour étudier la « réception » et l'adoption d'une posture d'extériorité à la situation par le chercheur constituent, en effet, un cadre d'interprétation qui structure la description. Il induit la mise en forme du contenu de l'observation et son orientation cognitive. Hélène et les garçons (TF1, 1992-94), par exemple, devient un moyen de confirmer la capacité des « séries collège " à intéresser, dans les années 90, les adolescents français scolarisés, et de la capacité de ces adolescents français scolarisés à s'identifier au monde mis en scène par les « séries collège ». La richesse empirique de l'observation menée par Dominique Pasquier est indéniable, notamment dans sa restitution de la fonction de sociabilité qui a contribué 
au succès commercial particulier d'Hélène et les garçons et dans la description de l'investissement affectif de ses personnages principaux par nombre de jeunes spectatrices. Mais l'interprétation revient à vérifier la conformation technique des personnages de la série aux personnes qu'elle doit toucher - une règle de l'industrie audiovisuelle - et l'identification des personnes touchées au sexe auquel elles ont été attachées - la fonction sociale de la famille - par leur éducation, bref la correspondance entre un savoir-faire technique éprouvé et un habitus sexuel préexistant. L'engagement corporel du spectateur que les discours et les observations en situation permettent de rendre sensible au lecteur ne constitue, au bout de la démonstration, qu'une confirmation du vecteur des représentations sociales que constitue la série télévisée et sa fonction de reproduction de l'ordre culturel. Du même coup, la position d'expertise singulière - résultant de son engagement corporel propre - du spectateur est sacrifiée à l'objectivation de la communauté d'interprétation à laquelle l'expertise sociologique de son discours permet de l'intégrer.

Cette limite est inhérente, de facto, à l'usage du public tout à la fois comme un objet observé et un principe d'interprétation sociologique. Elle conduit l'observateur de la consommation culturelle soit à vérifier l'appartenance de la personne à un groupe contrôlant ses réactions affectives (« l'ethnographie intégrative » où l'on va rapporter la conduite de l'individu à des normes collectives) soit à démontrer la qualification personnelle que l'individu retire de sa participation affective à une culture ( l'ethnographie narrative» qui décrira la construction de l'identité au sein d'une communautéf). Dans les deux cas, il y a une forme d'appauvrissement de la réalité observée, par la dissolution de la personne dans le collectif ou, inversement, par la réduction de la vie sociale à un vécu personnel. C'est ce que permet de corriger la pratique de l' "ethnographie combinatoire ${ }^{8}$ " proposée par les sociologues pragmatistes. Elle consiste à prendre en compte, justement, les effets inhérents au cadrage scientifique de l'observation, le chercheur s'incluant dans l'observation et s'efforçant d'identifier la manière dont les individus observés tirent profit de leur expérience pratique de la situation observée, des savoirs qui sont attachés aux situations et qu'ils peuvent mobiliser, même s'ils ne sont pas nécessairement aptes à les formaliser, pour (se) critiquer. Bref, c'est préférer se centrer sur la situation - l'acte de consommation - les réactions qu'il suscite, les évaluations qu'il entraine et les désaccords plutôt que sur le classement technique et social des individus qu'autorise leur discours sur la série. C'est être attentif, du même coup, à redonner au corps du spectateur son rôle d'instrument de mesure de «ce qui se passe» dans la consommation culturelle.

\section{Girls comme objet d'enseignement universitaire}

Utiliser Girls (HBO, 2012-17) comme le matériau d'un exercice universitaire ${ }^{9}$, c'est l'extraire du contexte de sa consommation ordinaire en même temps que sa temporalité commerciale. La première saison de Girls a bénéficié dès sa sortie, en 2012 d'une promotion par la critique française - en témoigne la présentation enthousiaste par Olivier Joyard de cette série dans Les Inrockuptibles ${ }^{10}$ - ou, en Australie, d'un battage médiatique («hype »), qui a conduit cette spectatrice à «voir ce qu'il en était» et à publier son jugement sur IMDB ${ }^{11}$ : 
So I thought I'd check « Girls » to see what the hype was all about. The storyline interested me, especially because it was about people my age. So far the positivity of this review. [...] I'm halfway through the first season now and I just turned it off because I couldn't watch it any longer. I've never seen such a bad show [...].

Les nombreux prix qui ont accompagné la sortie des six saisons, les débats lancés dans la presse féminine sur le manque de «correctness » de certains épisodes ont constitué, pour ceux qui les découvraient au fur et à mesure de leur programmation, un contexte médiatique qu'ignoraient les étudiants qui se sont vu proposer le visionnement de la première saison. Le nombre pléthorique de nouvelles séries aujourd'hui accessibles gratuitement sur les différents supports de diffusion offerts par la numérisation et son format court expliquent qu'aucun de ces étudiants, ou presque ${ }^{12}$, qui sont par ailleurs des consommateurs réguliers de séries de qualité13, n'aient eu l'occasion de la voir avant l'exercice de visionnement proposé. Si, par son horaire de diffusion sur les chaines de télévision françaises, la série Girls vise un public jeune - lycéen ou étudiant la reconfiguration des modes de consommation des séries empêche l'affectation d'un type de public à une série, ou un horaire. En effet, le public visé consomme en majorité des séries via des sites de streaming gratuit et des plateformes payantes type Netflix, plutôt qu'à la télévision. Peu d'étudiants sont abonnés à OCS par exemple. Le mode de fonctionnement spécifique de Netflix - le prix de l'abonnement étant indexé sur le nombre d'écrans, donc le nombre de personnes pouvant s'y connecter (sur le même créneau) permettant le partage de comptes avec ses amis, et un coût d'abonnement moindre - fait que les étudiants favoriseront ce type de plateforme plutôt qu'un abonnement à une chaine de télévision. Ne négligeons pas pour autant le poids de la diversité et de la qualité des programmes proposés, dans le choix des étudiants.

6 Ceci, joint à l'identité disciplinaire du cadre de l'échange - puisqu'il s'agit d'un cours d'études cinématographiques - constitue une modalisation de l'expérience cinématographique. L'obligation scolaire est de construire un discours sur un objet déterminé - «description de l'action » et " commentaire de la mise en scène ${ }^{14}$ " - et non de restituer ce que j'ai éprouvé, ce que cela m'a fait ou pas ${ }^{15}$. La longueur du propos a pour prix, dans les premiers textes produits, son caractère technique et l'attention à l'euphémisation, voire à la neutralisation de ses réactions personnelles. On note l'élision $\mathrm{du}$ " je » ou son usage avec un verbe épistémique (" je pense », " je crois ») et la revendication du caractère collectif de l'impression, « on », «nous ». Un exemple typique en est le commentaire suivant d'une étudiante :

C'est une série plutôt sympa à regarder, elle est bien filmée. On remarque qu'il y beaucoup l'utilisation du gros plan pour montrer les expressions des acteurs. De plus je trouve que la série parle bien de son sujet, chaque épisode se suit et ne s'égare pas de son sujet [sic]. Ce qui est assez sympa également c'est que nous savons qu'Hannah est le personnage principal de cette série, mais très vite on se rend compte que chacune des protagonistes a une importance, ce qui colle parfaitement avec son titre, "Girls». Je pense que des personnes, filles comme garçons, peuvent se reconnaître dans chaque personnage car ça traite du quotidien, des problèmes que l'on peut rencontrer à un moment de notre vie [...].

7 Lors de l'exercice sur la description de l'action, plusieurs étudiants parlent de voyeurisme, le fait que le spectateur serait "présent de manière intrusive", que «L'action dévoile un univers qui laisse recours au voyeurisme [parce que] le spectateur va être immiscé dans la vie des personnages comme si elle était la nôtre et tout est donné à voir. ». De la même manière, cette attitude semble révéler la tendance 
de ces étudiantes à traduire leurs impressions individuelles, leur gêne devant certaines scènes de la série sans doute, par une généralisation au spectateur.

Il s'agit d'une posture d'objectivation classique en études cinématographiques, celle de la réduction de l'expérience cinématographique à l'identification des qualités techniques de l'objet susceptibles de satisfaire le regard du spectateur. Gommant la participation corporelle de celui qui parle au spectacle dont il rend compte, elle s'oppose à la posture d'appréciation personnelle, de la spectatrice australienne déçue, qui intègre son corps - à la fois comme instrument de comparaison et comme moyen d'éprouver la justesse esthétique de la situation - dans sa reconstitution de l'expérience négative qu'elle vient de vivre :

I've never seen such a bad show. I should be able to relate to this. I myself graduated a year ago as well, taking my first steps on the job market, I have a boyfriend, I have great friends and I'm also struggling with « life " some times. But god, I am nothing like Hannah. She annoys me so much. I actually agreed with her parents during the first episode. You're 24 and you still need financial support from your parents to pay for rent? You dare to ask them 1.100 dollars a month? Spoiled brat. I'm 21 and I've been taking care of myself for over a year now [...]

I don't spend time with friends on the toilet, I don't want to shower with them, I can't even think of making jokes about rape during a job interview, if one of my friends had to undergo an abortion, I'd be a little more supportive, and I would NEVER be okay with a guy treating me like a piece of meat and being a total A-hole all the time. Adam is probably this type of character you need to give some time to get to know better, which is supposed to be interesting, but I just don't feel the need to watch another episode.

9 De fait, la manière dont la série touche l'intimité de celui qui la regarde par le caractère "graphique »des situations mises en scène n'est que suggéré dans le commentaire déjà citée l'étudiante, pour laquelle la série «nous apprend à rire et à ne s'inquiéter de rien, mais qui pourtant, nous montre des choses importantes». La possibilité de s'appuyer dans l'échange, écrit comme oral, sur une expérience partagée de la série - le visionnement de la première saison constituant la base du cours permet souvent de faire allusion à ces « choses » sans les identifier précisément et d'éviter, du même coup, à celui/celle qui parle de trop dévoiler son intimité. Ainsi, la liste des «choses importantes» - «les messages [...] sur le poids, les maladies, les premiers pas dans le domaine sexuel, une rupture, une rencontre, etc. » - énumère des sujets de discussion plutôt que les objets incongrus, des problèmes jusqu'alors «tabous » dans ce format de sitcom, qui l'ont marqué : Hannah, l'héroïne principale est grosse («le poids»), il s’agit de maladies vénériennes, de dépucelage, de masturbation, etc.

10 La situation d'échange universitaire ne facilite pas immédiatement, la valorisation de l'interpellation personnelle ressentie par le locuteur y compris lorsque celui-ci met l'accent sur la dimension "expérientielle » de la vision de Girls, le sentiment qu'elle « retranscrit bien nos luttes pour passer à l'âge adulte, ou du moins la perception que nous avons de cette étape de la vie ». Mais elle ne se manifeste pas moins :

Ce qui m'a marqué dans le premier épisode, c'est que les chocs de point de vue se faisaient par une rupture de l'intimité, les deux filles sous la douche, les deux filles aux toilettes, Adam et Hannah et leur relation sexuelle « bancale $»^{16}$.

11 L'évocation par le locuteur de ces " anecdotes réelles ${ }^{17}$ » qui lui offre l'occasion de sympathiser avec Lena Dunham, l'auteur de la série et principale interprète, nous 
signale la réflexivité que déclenche leur implication corporelle dans certaines situations mises en scène.

La mobilisation de la culture cinématographique n'empêche donc par la référence à une expérience personnelle de la vision et à ce qu'elle peut m'apprendre pour moi-même, et pas seulement pour apprendre à regarder des séries. Il s'agit de deux modalités inséparables de l'évaluation en situation d'une série, et la posture d'expertise technique ne peut en faire fi. La convergence des jugements des étudiants, leur accord pour reconnaître, non seulement le savoir-faire de la série - elle nous "attache " aux personnages -, mais la manière dont elle transgresse partiellement les normes techniques du format courant du sitcom, le confirment. Des textes produits à la vision des premiers épisodes, jusqu'au débat final, la mise en valeur de la place de la sexualité dans leur vie et la représentation crue qu'elle en donne singularisent incontestablement, pour les étudiants, la série Girls.

\section{L'expérience de la sexualité dans Girls et son ancrage dans le réel}

13 La dynamique de groupe engendrée par la réalisation de l'exercice et sa durée a fait ainsi s'accorder sur la « réalité » des questions posées par les interactions sexuelles mises en scène par la série.

14 La sexualité apparaît de manière récurrente, même centrale en réalité, dans la majorité des commentaires. L'avortement, le " VIH », le « dépistage », les « maladies sexuelles », le « sida » la virginité «tardive», la liberté sexuelle des femmes également. «La série semble aussi centrée sur les aventures sexuelles de chacun des personnages, les scènes sont explicites [...]». Liberté sexuelle qui est centrale dans le commentaire d'une étudiante chinoise, qui perçoit dans les personnages « quatre attitudes sexuelles dans la société américaine ", et qui voit en Jessa et en Shoshanna deux manières de " contrôler le droit de la liberté sexuelle des femmes ». On retrouve cette idée dans le commentaire de Madyson qui insiste sur l'aspect " purement sexuel» de la relation entre Adam et Hannah, sans pour autant juger négativement. «[...] Hannah couche avec un gars, une relation purement sexuelle. [...] relation drôle et purement physique. [...] Hannah [...] a une vie sexuelle libérée $[. .$.$] »; pour Julie " [...] les thèmes abordés sont plutôt féminins$ et jeunes : sexualité, grossesse, avortement, relation de couple, problèmes d'argent... »; Tom souligne la mise « $[. .$.$] en scène \left[\mathrm{d}^{\prime}\right]$ un spectre de problèmes comme l'incompétence de l'homme à se soucier de leur plaisir, la difficulté du dialogue générationnel, et une certaine absurdité du monde du travail. », pour lui :

Girls est une série qui cherche à mettre en image une la vision féminine, largement souvent sous représentée, de ces différentes femmes à une période charnière de la vie, entre l'adolescence et l'âge adulte.

On y suit des personnes comportant toutes leur lot d'excentricité cherchant à instaurer un pathos avec le spectateur. Mais le récit est surtout articulé autour du personnage d'Hannah, jeune femme à la recherche d'elle-même dans sa sexualité, dans sa volonté d'exister au prix de ses amis, et de s'affirmer au prix de ses parents.

15 Ál'opposé de la fin de non-recevoir que constitue la protestation morale de la spectatrice australienne justifiant son refus de la regarder au-delà du premier épisode, les étudiants admettent à la fois d'être concernés par ce que la série donne à voir et l'intérêt de l'ancrage dans le réel que produit sa vision. Une étudiante explique - 
d'abord en employant des termes personnels «ne me choquent pas» ou «ce qui me répugne ", puis par une euphémisation en parlant du «spectateur », puis par l'emploie $\mathrm{du} \ll$ on $»:$

Dans la série Girls, ce qui choque le plus dans les scènes de sexe est vraiment le fond. Comme Marnie qui n'aime pas les relations sexuelles qu'elle a avec son copain mais elle ne lui dit rien. Mais cela est sûrement fait pour faire réfléchir le spectateur sur la sexualité dans la vie de tous les jours, sur les problèmes sexuels, etc.

De la même manière, une autre étudiante estime que la série «casse les tabous » en montrant différents types de rapports sexuels, qu'elle juge "parfois un peu maladroit (mais au final, la sexualité n'est-elle pas maladroite ?) ». Elle transforme, pour chacun, la sexualité en matière à réflexion par le biais de l'exploration qu'il opère avec son propre corps des interactions imaginées par l'auteur. De ce fait, le matériau émotionnel que mobilise la série, « la peur du sida ", le déplaisir de «se faire surprendre lors d'une conversation intime ", le caractère troublant de certains " gestes sexuels explicites ", le malaise devant des situations «loufoques (type masturbation féminine/masculine sortie de nulle part, harcèlement sexuel qui transforme presque Hannah en harceleuse à son tour...) ", la confusion entre certaines positions sexuelles « les scènes de sodomie [qui sont en réalité des levrettes] sont les plus gênantes et les plus nombreuses.» ouvrent à une interrogation de sa propre conduite sexuelle. Cette interrogation personnelle, ce rapport de soi à soi que permet d'opérer la fiction doit, pour s'exprimer dans le contexte de notre observation, composer avec le discours d'objectivation du caractère fictif du récit qu'entraîne la dynamique de groupe créée par le cours. Comme dans le cas de l'observation par Sabine Chalvon-Demersay de la réception de la série française L'Instit (France 2, 1993-2005), la dynamique conversationnelle lorsqu'on interroge longuement le réalisme de la série conduit naturellement à la « défictionnalisation » du regard porté sur elle ${ }^{18}$. Un étudiant souligne donc, avec le recul, que si « l'image très forte, avec un peu d'absurdité et de gêne » que donne la série de la sexualité « sert son propos, qui est de rappeler la notion de plaisir (surtout celui de la femme)", ce qui est généralement "tabou et passé sous silence», elle manque d' « objectivité » car « toutes les relations se ressemblent dans la série, ce qui n'est pas le cas dans la vraie vie ». Ou bien, comme l'exprime plus prosaïquement cette autre étudiante :

DU HAUT DE MES PETITS 22 ANS (sic), je trouve que ces scènes restent tout de même romancées. Effectivement, certaines situations arrivent, mais que chaque partie de jambes en l'air se clôture par une blague ou quelque chose d'extraordinaire bloque peut-être le fait de totalement se reconnaître dans les personnages. Toutefois, cela reste une série, et ce «petit » reproche s'applique à presque tous les films/séries.

17 On retrouve la même idée dans le commentaire d'une autre étudiante :

Je trouve que les scènes à caractère sexuel sont un peu trop présentes. La série essaie de nous montrer le quotidien des personnages, alors que dans la vraie vie, la sexualité n'est généralement pas omniprésente. Les scènes ont cependant le mérite d'être plutôt réalistes, plus en tous cas que dans la plupart des séries du genre, où elles sont souvent romancées. On voit par exemple les personnages parler [pendant l'acte], mettre un préservatif ou encore se faire mal durant l'acte, ce qui est rare à l'écran.

18 De ce fait, l'engagement que font les étudiants des images de la sexualité donnée par la série s'effectue selon deux registres différents, celui de leur mise à l'épreuve personnelle par le contenu et l'orientation de certaines scènes représentées, et celui de 
leur évaluation de la manière dont la série peut « jouer un rôle dans la transformation des représentations sociales et les dynamiques de changement social et culturel ${ }^{19} »$.

\section{Apprendre de l'expérience de la vision de Girls}

19 Être marqué par la présence de la sexualité dans Girls, c'est à la fois s'y rendre sensible et s'en faire le porte-parole sur la base de la «familiarité en personne »que l'expérience, au sens de notre compétence de spectateur, nous permet de nouer avec les personnages, même s'ils sont fictifs. Philippe Descola, appelle «ontologie animiste » la posture cognitive qui nous permet d'éprouver réellement une continuité morale avec des êtres non-humains, qui sont de simples images, et de commercer avec eux. C'est ce qu'exprime l'étudiant qui pointe les «émotions autres que le plaisir sexuel » que suscite "la masturbation solitaire d'Adam», ou le "coït des parents d'Hannah sous la douche » ou « la personnalité extravagante d'Hannah quand elle tente de lui [son partenaire] mettre un doigt dans le cul alors que lui cherche une relation plus classique». Ou encore lorsqu'il signale son acceptation de «ce qui peut en déranger certains », " par exemple pour Jessa qui souhaite être libre et donc va avoir une relation en « coup de vent » avec un ex-copain [...]» ou :

[...] pour Marnie et la relation qu'elle a avec son ex-copain si l'on peut dire. Durant l'acte, elle semble encore plus perdue qu'elle ne l'était déjà. A savoir, vouloir une relation amoureuse et donc tout faire pour garder son copain, mais aussi le fait qu'elle commence à comprendre qu'elle ne l'aime pas et finira par lui dire en plein acte.

Rire de ce qui arrive à l'écran en acceptant d'y participer, et accepter de penser avec le personnage même si ce qu'il fait nous est étranger est la manière dont la série permet d'avoir une expérience qui nous éclaire sur nous même et autrui.

Les conservations engendrées par les «traumatismes" rendent bien compte du caractère positif, d'un point de vue éthique, du passage de l'implication personnelle dans la scène à la relativisation de la fiction. Le cas d'une étudiante qui est marquée par une scène de relation sexuelle est très éclairant. Dans cette scène (S01E01) Hannah rend visite à Adam, qui au milieu d'une discussion ordinaire, lui explique que dans la vie, elle ne doit être l'esclave de personne. Sauf le sien. Hannah se jette alors dans ses bras, et les deux amants entament les préliminaires. Adam demande à Hannah de se mettre sur le ventre, il lui indique ensuite toutes les positions à adopter, Hannah s'exécute. Bien que cette situation soit parfaitement consentie - il ne s'agit en aucun cas d'un viol - et que lors de la projection de cet épisode, les rires dominent parmi les réactions des étudiants, le comportement d'Adam, qui «traite mal Hannah» est perçu comme violent, humiliant par cette étudiante. Elle conservera une animosité pour le personnage d'Adam jusqu'à la fin de la saison, dénonçant de manière récurrente sa manipulation du corps d'Hannah:

J'ai [...] été irritée par quelques scènes de sexe dans les premiers épisodes que je trouvais très sexistes, le garçon disant à Hannah ce qu'elle devait faire et lui interdisant de jouir. [...] J'ai personnellement vue cette scène presque comme un viol banalisé. Je trouve cependant que ce sexisme est en soi un certain réalisme de la sexualité aujourd'hui où le plaisir des femmes est souvent mis au second plan. Je trouve cela dommage que les personnages de la série ne trouvent pas ça problématique. 

amènera un étudiant à réagir :

J'ai l'impression que tu passes complètement sur le fait que c'est montré d'une certaine manière. Ils auraient voulu dire «ouais c'est un propos normal », ils auraient fait des plans sur le mec qui est en train de prendre du plaisir et enfin voila ce qui est montré et complètement mis en avant dans cette scène, comme tu disais déjà c'est bien de le montrer parce que effectivement des filles qui couchent avec des mecs sans prendre de plaisir et qui acceptent des trucs qu'elles ont pas envie, tout ça, ça existe, il faut le montrer. [...] Mais on sent que dans cette scène, justement, on s'attarde vraiment sur le visage de Hannah [...] le propos c'est pas de dire " ha faut faire ça », le propos c'est de dire "ça existe et elle est mal à l'aise ", on la sent pas heureuse ni rien, on voit vraiment son visage mal à l'aise, elle ose pas trop dire quelque chose parce qu'effectivement elle a l'air plus ou moins amoureuse du personnage, elle est un peu, c'est son point de vue qui est mis en avant, c'est pas le fait que cette pratique est normale, c'est plus le fait de dire « regardez, elle est en train de pas dire non à un truc qui pourtant la gêne quand même ", c'est ça qui est mis en avant [...].

La relativisation, on le voit, ne conduit pas à annuler l'ancrage de la scène dans la réalité, mais à accepter l'incitation à réfléchir sur l'expérience de la sexualité qu'elle nous adresse. Ce qui est une technique du corps - le spectacle en tant que jeu, en tant qu'exercice de la fiction - devient une " technique de soi ", une manière d'interroger sa propre subjectivité, de découvrir ses limites, d'interroger «ce qu'il.elle trouve acceptable ou pas». Ce qui advient souvent pour le spectateur «à son corps défendant », dès lors que sa participation au spectacle le conduit à opérer un retour sur soi, et à interroger son propre désir :

Adam et ses fantasmes sont assez perturbants. La scène qui m'a le plus choqué avec lui est la scène où il se masturbe devant Hannah et que celle-ci se prend au jeu. Il ne s'agit que de personnages fictifs, mais le caractère d'Adam me perturbe.

Faire confiance à son expérience, c'est reconnaître notre incertitude par rapport au désir d'autrui, que, par exemple « l'on ne comprend pas pourquoi Hannah retourne voir Adam alors qu'il se sert d'elle, mais l'utilise comme un objet pour satisfaire ses besoins». C'est admettre en même temps qu'Adam «assume ses fétiches et ses fantasmes et personne n'a le droit de le juger ou de le mépriser » dès lors qu'il a «le respect de ses partenaires ». Bref, prendre au sérieux la fiction cinématographique, qui aide ici à le faire en traitant avec humour les choses sérieuses ${ }^{20}$, est saisir l'occasion d'adopter un point de vue éthique, «d'évaluer ce que nous faisons, ce que nous disons, d'après le mode d'existence que cela implique " plutôt que de juger des actions et des intentions en termes de bien et de mal, posé comme des valeurs transcendantes ${ }^{21}$. L'adoption de cette posture éthique est cela même qui permet de ressentir les limites de légèreté dont font preuve les personnes représentées, dès lors qu'elles ne prennent pas soin d'elles-mêmes et d'autrui - le cas de l'avortement (S01E02) - où acceptent d'être harcelées sexuellement.

\section{Une série « féministe »?}

Le caractère marquant de la place centrale que Girls donne à la manière dont des jeunes femmes vivent leur sexualité est un point d'accord entre l'ensemble des contributions écrites et des prises de parole. Un accord à la fois intellectuel et émotionnel, puisque «faite pour faire réfléchir les jeunes adultes qui sont en pleine recherche de 
leur sexualité » elle "montre que tout le monde vit la même chose », quel que soit le sexe du spectateur. C'est cette sympathie émotionnelle partagée malgré la différence sexuelle qu'exprime de façon heureuse cette formulation - presque comique, puisqu'elle ne respecte pas la «logique logique» - par un étudiant de son impression : «ce que veut montrer la série Girls, c'est que le personnage principal nous ressemble, plus particulièrement aux femmes ". Sa valeur, du point de vue d'un spectateur homme, est du même coup qu'elle « nous permet de mieux comprendre les femmes et leurs motivations ». Cette confirmation pratique du " caractère polymorphe de l'identification » cinématographique selon Edgar Morin ${ }^{22}$ lui permet de reconnaître le savoir-faire de la série : en effet, « Hannah est un personnage complexe » et " grâce à Hannah et ses amies, le spectateur a [sic] une multitude de personnalités différentes mais aussi de récits différents ». Mais le plaisir procuré par ces identifications multiples achoppe sur la valorisation du ressenti féminin des situations. Même si les personnages masculins « ont un rôle très important » ils ne sont " pas assez complexes, devenant même trop lisses à côté d'Hannah ».

La dynamique du forum - la dernière séance du cours, consacrée à une évaluation collective de l'intérêt de la série - enclenche ainsi une «montée en justice» des participants dans l'expression de leur jugement définitif sur sa nouveauté. Ceci s'observe particulièrement lorsqu'il s'agit d'évaluer, au delà de sa valeur documentaire, la contribution de la série au progrès du féminisme. Ainsi leur accord tendanciel dans leurs contributions écrites pour caractériser la série comme " progressiste » dans la manière dont elle met en scène le comportement des jeunes femmes, notamment en matière sexuelle - «chaque femme est libre de son corps!»- laisse la place à des désaccords explicites sur le jugement à porter sur cette série dans le cadre de l'espace public. Ce jugement se construit différemment en effet selon que celui qui parle vise à confirmer à d'autres consommateurs expérimentés sa compétence à expertiser l'objet donc on parle ou qu'il s'inscrit dans une situation de transmission d'une expérience à des personnes qui ne l'ont pas vécue. L'explicitation argumentée, ex post, de la manière de reconnaître ou contester la dignité technique ou sociale de l'objet laisse la place à la parole prudente. Elle est celle du consommateur-témoin qui s'exprime à la fois en tant que citoyen et en tant que personne, et perçoit la nécessité de tenir compte de la nécessité de relativiser la perception des qualités de la série en fonction de la situation (seul(e), avec des ami(e)s, en famille ${ }^{23}$, etc.) et de la manière de s'y engager personnellement. Ceci légitime que l'on puisse, «en tant que femme», contester l'adéquation de la qualification de féministe à la série, parler d'un "vernis féministe " car « je la trouve à la limite assez fausse sur la question de l'avortement ou du harcèlement au travail » ou encore le rappel à la prudence, «sur la sexualité je trouve que c'est féministe, sur d'autres non il y a différentes formes de féminisme ». Il est notable que ce « en tant que femme » n'est pas attaché à la personne qui parle, mais à la situation. C'est ce qui rend possible pour certains étudiants d'adopter la posture du féminisme militant, comme cet étudiant maîtrisant bien le discours sur l'image qui n'hésite pas à affirmer «la grosse erreur de Girls c'est de montrer des filles qui composent avec le jugement de la société ». Ce jugement péremptoire entraîne, de la part aussi bien d'étudiants que d'étudiants, un rappel à la réalité, à la fois technique et sociale, car « elles ne savent pas nécessairement ce qu'elles veulent » et, dans le cas du personnage de Marnie mis en cause par l'étudiant féministe, «c'est aussi elle et son choix personnel, il n'y a pas que la société », les personnages sont des «femmes 
normales [...] la fille qui couche avec son mec pour pouvoir le garder, cela arrive constamment ».

Ce chassé croisé qui brouille les assignations identitaires confirme l'impossibilité d'attacher a priori à un spectateur un type de conduite sans en passer à la fois par l'action du spectacle, par la manière dont il s'y rend sensible personnellement et la forme de sociabilité qu'il noue par son intermédiaire. Un étudiant peut ainsi manifester une sensibilité « féminine » à la différence revendiquée par le corps d'Hannah - alors que Monica dans Friends «a des problèmes avec son poids" - et une étudiante l'extériorité "masculine » de celui qui n'a pas à se poser la question, même si «c'est vrai qu'elle a des rondeurs $^{24}$ ». Il en va de même pour l'antipathie et la sympathie « sociale ». Pour l'ensemble du groupe, Girls ne met absolument pas en scène leur milieu social ${ }^{25}$ - «nous, c'est la galère par rapport à eux, eux, c'est le haut du panier ", « une élite WASP ", « un milieu petit-bourgeois » - ce qui n'empêche pas la plupart d'entre eux de sympathiser avec «eux», et d'apprécier le savoir-faire d'une série capable de les «concerner», «je me suis senti concerné par certains personnages ».

L'observation du forum confirme la nécessité d'éviter de «sursocialiser »ou, à l'inverse, de « sous-socialiser » la conduite des consommateurs de série, en faisant fi de la manière dont ils réfléchissent leur engagement personnel dans la situation de consommation et dans les situations mises en scène. Ceci s'applique également à « l'émergence de héros perfectionnistes de type nouveau » justement relevée par Sandra Laugier dans les séries contemporaines ${ }^{26}$. Cette émergence ne se laisse pas réduire à la mise en forme de personnages ayant une fonction de réparation d'une image négative, ce qui les réduirait de facto à de purs symboles. C'est dans l'interpénétration de leur capacité, à l'écran, et de la nôtre, dans la salle, à « " avoir une expérience ", à en tirer des formes et des forces de vie et à s'éduquer dans la vulnérabilité même, physique et morale... » que réside leur caractère marquant. Ceci permet de relativiser une analyse en termes de " héros » indifférente à cette dimension de l'engagement personnel du spectateur et à son expertise de consommateur.

À ce titre, le rapport que les étudiants et les étudiantes entretiennent avec le personnage d'Adam est édifiant ${ }^{27}$. Son comportement machiste à l'égard d'Hannah provoque d'abord une réserve, sinon, un rejet. Alors qu'ils le percevaient unanimement comme «lourd», «farfelu», « idiot », « immature», il figure, au terme de la saison, parmi les personnages les plus appréciés des filles comme des garçons. Les qualités humaines qui lui sont désormais reconnues - la "sincérité ", l'attention, la générosité ainsi que ses faiblesses - son côté "fragile ", "son passé difficile " lié à la découverte (S01E07) de son passé d'alcoolique, qui se bat aujourd'hui contre son addiction -, contrecarrent son côté "obsédé sexuel», "pervers", «bizarre», et le rendent « terriblement humain» au fil des épisodes. Il a " évolué de manière positive pour les spectateurs ", tout comme la meilleure amie d'Hannah, Marnie, d'abord rejetée pour son manque de respect vis-à-vis d'elle-même. Sa sollicitude à l'égard d'autrui emporte l'estime, finalement, de certaines étudiantes: «J'aime le fait qu'elle soit là pour ses amies [...] », « Elle est gentille, a de l'empathie [...] », «[...] elle me ressemble sur pas mal de sujets, notamment sur sa gentillesse, elle donne beaucoup aux autres. $[\ldots] »$. 


\section{Ordinaire de la série et personnage " perfectionniste »} spectatrices d'Hélène et les garçons - qui révèlent qu'à $67 \%$ elles préfèrent, tout comme les hommes, les personnages masculins - par la formule «On aime les personnages masculins, mais on parle aux personnages féminins». Il s'agit d'une confirmation du conformisme social du choix, la vérification de la domination masculine, à la fois symbolique et sexuelle :

Il est intéressant sur ce point de vérifier que les choix des filles se portent plus fréquemment sur un personnage féminin lorsqu'il s'agit de citer le personnage qu'elles aiment le moins: les critiques qu'elles avancent à leur encontre corroborent d'ailleurs l'hypothèse de la relation amoureuse (on critique Laly parce qu'elle fait marcher les garçons, ou Tiffany parce qu'elle est égoïste et superficielle $\left.{ }^{28}\right)$.

31 Si l'on admet la valeur documentaire de l'enquête réalisée par Dominique Pasquier alors notre observation confirme la nécessité de prendre la mesure, à la fois de l'évolution de la mentalité du public féminin, de la consommation «télévisuelle » (devenue pléthorique du fait de sa numérisation) et de la manière d'étudier la réception. étudiants et étudiantes avec lesquels nous avons travaillé invite à des conclusions plus nuancées.

Si l'attachement à une série en passe par les personnages, on ne peut réduire l'attachement constaté ex post à une série à un personnage. La performance de l'acteur joue un rôle, difficile à exprimer dès lors que l'expérience de cette performance fait communiquer, du point de vue du ressenti du spectateur, l'interprétation de l'acteur et sa participation en personne, sa présence. C'est ce qu'illustre Clément, incapable de nommer ses acteurs et actrices préférés parmi l'ensemble des personnages qui constituent le monde mis en scène par une série :

Certes, j'aime beaucoup ce qu'ils font, et j'en apprécie plus que d'autres, mais je ne pense pas pouvoir en sortir un ou deux du lot comme je les aime tous. Je pourrais dire JCVD, mais ce n'est pas tant l'acteur qui me plaît, mais la personne qu'il est dans la vie.

«La personne qu'il est dans la vie » désigne ici plus la «formule personne-rôle », selon Goffman - la capacité d'une personne à donner l'impression qu'elle convient au rôle que le rapport «rôle-personnage ", c'est-à-dire l'engagement personnel de l'acteur dans la vie social ${ }^{29}$. Une autre étudiante, Lorraine, valorise explicitement ces deux dimensions de la performance. Emma Watson figure parmi ses actrices préférées « pour ses engagements » au côté d'Eva Green qui y figure " pour ses rôles ». On retrouve cette attention à ces deux dimensions de la performance dans le discours de Benjamin, lorsqu'il décrit son admiration pour Emma Watson : «j'étais amoureux d'elle + femme incroyable + elle utilise sa notoriété pour le féminisme et c'est vraiment cool.», l'attraction sexuelle qu'il a ressentie faisant partie de sa performance d'actrice.

Cette capacité à discriminer intuitivement les cadres de l'expérience sans nécessairement formaliser leur jugement sur ce point, en même temps que la très grande diversité des êtres humains et non-humains avec lesquels ils commercent 
différencie en ce sens notre observation d'autres études de réception. Le fandom, au sens de la promotion exclusive d'un acteur ou d'une série n'est pas observable chez les étudiants et étudiantes. L'actrice-fétiche mis en avant par une des étudiantes n'est qu'une des interprètes que sa consommation des séries depuis l'adolescence lui a permis de découvrir. Et, là encore, ce n'est pas seulement son personnage, mais l'engagement éthique de l'actrice - Eliza Dushku, qui incarne, entre autres, le personnage de Faith dans Buffy contre les vampires (The WB, 1997-2003) - qui explique la tendresse particulière qu'elle éprouve vis-à-vis d'elle, et son plaisir lorsqu'elle revoit Faith (l'étudiante mêle le nom de l'actrice et le nom du personnage) :

[...] je dois avouer que pour la plupart j'aime ces acteurs ou actrices parce que j'ai aimé leur personnage à l'écran et du coup inconsciemment je me suis aussi accrochée à eux parce que pour moi personnage et acteur sont en lien (ils ne forment presque plus qu'un). Par contre pour Eliza Dushku, j'ai appris à connaître l'actrice, j'ai même pu la rencontrer au Comic-Con de 2016 et discuter avec elle. Eliza Dushku correspond parfaitement à l'image qu'elle renvoie sur les réseaux sociaux. C'est une femme engagée, activiste, qui défend ses projets, qui défend les gens, qui est intéressée par les autres. C'est ce qui a renforcé mon attachement à elle, vis-à-vis de Faith.

L'échange avec les étudiants et étudiantes confirme donc leur réflexivité et leur capacité à gérer les deux cadres de l'expérience de consommation d'une série. Leur goût pour leur série est un "faire", une activité de consommateur et consommatrice averti de cinéma, mesurant leur satisfaction à l'aune de critères de qualité du spectacle, à commencer par l'interprétation telle qu'ils et elles l'on éprouvée :

Mes actrices préférées : Emilia Clarke, Charlize Theron, Jennifer Lawrence, Elizabeth Moss,... (liste non-exhaustive). Emilia Clarke [Daenerys dans Game of Thrones] particulièrement pour son jeu d'actrice vraiment remarquable (« jouer sur un dragon » où elle doit imaginer qu'il est là, apprendre des pages entières en Dothraki et Valirien (des langues qui n'existent pas). Je les admire pour leur qualité de jeu, pour leur capacité à incarner des rôles souvent difficiles, mais elles parviennent totalement à nous faire adhérer à leurs personnages.

Pour Alice, il ne s'agit cependant que d'un critère de qualité, même s'il est crucial et lui permet de s'orienter dans l'offre de visionnement ${ }^{30}$. Le nom des interprètes, conjointement avec l'indication du genre ( si ce sont des thématiques qui me plaisent ») et la renommée de la série («si j'entends beaucoup parler de la série je vais regarder 1 ou 2 épisodes pour voir si j'accroche ou pas ») sont autant de justifications de la tester.

L'épreuve de la série, dans la mesure où elle est celle de sa capacité à faire tenir ensemble une histoire, des interprètes, une mise en scène, une musique est, pour tous, déterminante. Comme l'indique Camille :

Je trouve cette série [American Horror Story (FX, 2011-)] fascinante. Les histoires étranges, glauques, malfaisantes, le montage, les musiques, les jeux d'acteurs. [...] Pareil pour Hannibal [NBC, 2013-15], les acteurs, le montage, les histoires, la psychologie du personnage de Lecter. Bien plus proche du bouquin que des films (du coup j'ai lu les livres pour mieux comprendre l'histoire).

Lorraine, une grande consommatrice de séries, peut ainsi les comparer en pointant leurs points forts au regard de la maîtrise de ces savoir-faire. Elle aime :

Love, Death [and] Robots [Netflix, 2019-] pour tous les types d'animation qu'on y trouve [...] Stranger Things [Netflix, 2016-] pour l'ambiance et l'intrigue [...] Games of Thrones pour sa quantité d'intrigues et de personnages; Peaky Blinders pour le visuel ainsi que les personnages [...] La Forêt de l'étrange [Cartoon Network, 2014] car 
l'étrangeté intrigue beaucoup et les personnages sont touchants [...] Glow [Netflix,

2017] pour les personnages et les enjeux.

la vision de la série s'ancre, pour les étudiants et les étudiantes interrogées, dans l'espace public et ses échanges artistiques et politiques. Les étudiantes déclarent ainsi être concernées par des personnages féminins forts, comme Lorraine qui apprécie «Brienne of Torth car c'est une femme qui rejette ce que la société attend d'elle (être une lady) en étant chevalier (Game of Thrones) ", ou Célia dont les actrices préférées sont Emilia Clarke et Sophia Bush parce qu'« elles incarnent toutes les deux le modèle de la femme forte et indépendante qui se relève toujours et qui n'a besoin de personne ». Julie intègre dans ses séries préférées The Bold Type [Freeform, 2017-] et Good Girls [NBC, 2018-] parce qu'elles «mettent en avant trois femmes fortes et indépendantes mêlant humour et drame." Elles s'expriment ainsi en tant que spectatrices, représentantes d'un public féminin, qui n'aspire pas simplement à l'augmentation de l'«offre de personnages féminins ", mais exprime une exigence perfectionniste. Si, pour Camille, Supernatural [The WB, 2005-] est l'une des séries contemporaines qui l'ont le plus marqué, elle regrette tout de même le caractère « inexistant » des personnages féminins dans leur relation aux hommes :

[...] elle me fait penser à Buffy, mais avec une vision masculine. Cependant, je la trouve de plus en plus « sexiste " au fur et à mesure. Il n'y a pas de personnages féminins forts, et lorsqu'il y en a une, elle meurt. Au lieu de rajouter des femmes à la série, ils ont créé une série parallèle avec que des femmes... Je trouve l'idée très nulle.

C'est la juste attention donnée aux personnages féminins par Game of Thrones qui justifie, au contraire, le plaisir pris par Alice à cette série et le progrès auquel elle contribue. En effet, elle « regorge de personnages féminins extrêmement bien écrits et riches. Ce qui à mon opinion manquait cruellement jusqu'ici. »

\section{Conclusion : Les limites de l'interprétation?}

Les caractéristiques socioprofessionnelles du lieu de notre observation et la nature de son public limitent évidemment le contenu de notre observation. Á l'inverse d'une vulgate sociologique courante, il nous semble cependant qu'elle rectifie un biais, inhérent à l'adoption par le chercheur d'une position d'extériorité par rapport à ce dont il parle, qui lui interdit d'avoir une position équitable par rapport à l'observé. Elle lui impose en effet au nom de la nécessité d'objectiver son rapport à l'objet de faire fi, non seulement de sa propre implication dans la consommation culturelle qu'il observe, mais de l'expérience qu'en retire ceux qu'ils observent $\mathrm{Si}$, comme nous l'avons souligné, le cadre de notre observation pèse sur l'expression des personnes rien ne justifie que l'on doive réduire le contenu de leur expérience de consommateur à l'exercice de l'habitus technique - parler du cinéma - qu'ils doivent incorporer en tant qu'étudiants. Intégrer dans notre analyse leur savoir commun de spectateur et la sociabilité qu'ils 
entretiennent avec les objets dont ils parlent est ici une manière de comprendre, au lieu de leur refuser a priori, leur capacité à se perfectionner.

\section{BIBLIOGRAPHIE}

CHALVON-DEMARSAY, Sabine, «Enquête sur des publics particulièrement concernés. La réception de L'instit et d'Urgences ", in Les Sens du public : publics politiques, publics médiatiques, éd. Daniel CÉFAÏ et Dominique PASQUIER, Paris, PUF-CURAPP, 2003, p. 501-521.

DELEUZE, Gilles, Pourparlers. 1972-1990, Paris, Minuit, 1990.

DODIER, Nicolas, BASZANGER, Isabelle, « Totalisation et altérité dans l'enquête ethnographique ", Revue Française de Sociologie, vol. 38-1, janvier-mars 1997, p. 37-66, https://www.persee.fr/doc/ rfsoc_0035-2969_1997_num_38_1_4571.

ESQUENAZI, Jean-Pierre, « David Kelley et la politique de la complicité », Mise au point, n 3, 2011, https://journals.openedition.org/map/969.

GOFFMAN, Erving, Frame Analysis. An Essay on the organization of Experience, New York, Harper and Row, 1974.

HENNION, Antoine, Les professionnels du disque. Une sociologie des variétés, Paris, Métailié, 1981.

JOYARD, Olivier, « "Girls" : la série du printemps », site des Inrockuptibles, 12 mai 2012, https:// www.lesinrocks.com/series/girls-la-serie-du-printemps-5340-12-05-2012/.

JULLIER, Laurent, LEVERATTO, Jean-Marc, « Delon and Performance: Emploi and the Interaction between Individual, Role and Character ", in Alain Delon. Style, Stardom and Masculinity, eds. Nick REES-ROBERTS and Darren WILSON, New York, Bloomsbury Academic, 2015, p. 31-42.

LAUGIER, Sandra, «Présentation. L'autre voie de la philosophie morale », in La voix et la vertu. Variétés du perfectionnisme moral, éd. Sandra Laugier, Paris, PUF, 2010, p. 1-30.

MADELRIEUX, Stéphane, « Expériencer », Critique, n 787, décembre 2012, p. 112-113, https:// www.cairn.info/revue-critique-2012-12-page-1012.htm.

MANN, Brenda, SPRADLEY, James, Les bars, les femmes, la culture [1 $1^{\mathrm{e}}$ éd. : 1975], Paris, PUF, 1979.

MORIN, Edgar, Le cinéma ou l'homme imaginaire, Paris, Minuit, 1952.

MORIN, Edgar, Les stars, Paris, Seuil, 1957.

PASQUIER, Dominique, « "Chère Hélène". Les usages sociaux des séries collège », Réseaux, vol. 13, n 70, 1995, p. 9-39, https://www.persee.fr/doc/reso_0751-7971_1995_num_13_70_2665.

QUÉRÉ, Louis, « Faut-il abandonner l'étude de la réception ? Point de vue », Réseaux, vol. 14, n 79, 1996, p. 31-37, https://www.persee.fr/doc/reso_0751-7971_1996_num_14_79_3779. 


\section{NOTES}

1. Un exemple princeps en est l'étude pionnière en France de réception de la série télévisée d'Hélène et les garçons réalisée par Dominique Pasquier, dans laquelle cette série sert de moyen d'objectivation de « l'usage social » des « séries collège ». Cf. l'article séminal, «"Chère Hélène". Les usages sociaux des séries collège ", Réseaux, vol. 13, n 70, 1995, p.9-39, https:// www.persee.fr/doc/reso_0751-7971_1995_num_13_70_2665.

2. Un bon exemple en est l'objectivation des «moyens de retenir l'attention des publics et de définir l'identité de la série "perceptibles dans les séries écrites et produites par un "showrunner" américain réputé, David Kelley. Cf. Jean-Pierre Esquenazi, « David Kelley et la politique de la complicité », Mise au point, n 3, 2011, https://journals.openedition.org/map/969.

3. Antoine Hennion, Les professionnels du disque. Une sociologie des variétés, Paris, Métailié, 1981.

4. Henri Bergson signalait déjà la supériorité philosophique, pour décrire cette conduite, de la langue anglaise, qui possède le verbe « to experience», qu'il faudrait traduire par « expériencer »; voir Stéphane Madelrieux, «Expériencer », Critique, n 787, décembre 2012, p. 112-113, https://www.cairn.info/revue-critique-2012-12-page-1012.htm.

5. Louis Quéré, «Faut-il abandonner l'étude de la réception ? Point de vue », Réseaux, vol. 14, n 79, 1996, https://www.persee.fr/doc/reso_0751-7971_1996_num_14_79_3779, p.34.

6. Ibid., p. 36.

7. Nicolas Dodier et Isabelle Baszanger, «Totalisation et altérité dans l'enquête ethnographique », Revue Française de Sociologie, vol. 38-1, janvier-mars 1997, p. 37-66, https:// www.persee.fr/doc/rfsoc_0035-2969_1997_num_38_1_4571.

8. C'est-à-dire qui adopte comme principe de se focaliser sur la situation et d'examiner les variations des formes de coordination entre les personnes et les objets au sein de la situation observée. Ibid.

9. Le cadre de l'étude était le TD «Étude d'une œuvre ou d'un cinéaste » dispensé en $3^{\mathrm{e}}$ année de licence « Arts du spectacle et audiovisuel, spécialité cinéma » de l'UFR Arts, Lettres et Langues de l'Université de Lorraine, site de Metz, animé par Elodie Valkauskas. Lors de chaque séance de deux heures, deux épisodes de la première saison de Girls étaient projetés aux étudiant.e.s. Ces projections ont donné lieu à des productions écrites (anonymes ou non) à partir de questions posées (le sujet, la mise en scène, la représentation de la sexualité, le rapport avec les personnages) et à des échanges oraux. La dernière séance a donné lieu à un échange collectif sur la série, à partir de propos relevés dans des critiques parues dans la presse française sur la série Girls. Sur le modèle de l'enquête de Brenda Mann et James Spradley, Les bars, les femmes, la culture [1 $1^{\mathrm{e}}$ éd. : 1975], Paris, PUF, 1979, des entretiens réguliers entre l'animatrice et Jean-Marc Leveratto ont servi à préciser des aspects des échanges perçus et ressentis mais difficiles à formaliser.

10. Olivier Joyard, «"Girls" : la série du printemps », site des Inrockuptibles, 12 mai 2012, https:// www.lesinrocks.com/series/girls-la-serie-du-printemps-5340-12-05-2012/. La série était en effet accessible aux spectateurs français, dès sa sortie aux USA en 2012, sur VOD Orange.

11. "I'm in my early 20s and I don't like this show » par Kimvanwuytswinkel, publié le 3 octobre 2015, www.imdb.com/review/rw3328005 (consulté le 2 avril 2019).

12. Selon une consultation à la cantonade, avant de commencer l'expérience, deux étudiantes avaient vu la série.

13. Game of Thrones (HBO, 2011-) est la série qui apparaît avec le plus de récurrence parmi les séries préférées de cette promotion. Émergent ensuite Peaky blinders (BBC Two, 2013-), Breaking bad (AMC, 2008-2013) et Friends (NBC, 1994-2004).

14. Telles étaient les consignes du $1^{\mathrm{e}}$ et du $2^{\mathrm{e}}$ exercice proposé.

15. «Personnellement, si l'on ne m'avait pas forcé à le regarder j'aurais abandonné le premier épisode au bout de cinq minutes ». Pour une petite frange des étudiants férus d' « image » (savoirfaire technique) et d'" action ", la série est, en tant que sitcom, immédiatement disqualifiée 
car « dure à suivre [...] il y a beaucoup (trop) de dialogues à mon goût. C'est très vite fatigant pour moi et je décroche régulièrement dans ce flot de blablabla ».

16. On note l'interpénétration dans la formulation entre la description de ce qui est montré et ce qui est ressenti par le spectateur, la « rupture de l'intimité » notamment, pour désigner les scènes où les protagonistes partagent la même salle de bain et se douchent ou urinent sous le regard de l'autre.

17. La formule exprime de façon économique ce qu'exprime plus longuement une autre contribution anonyme: «je trouve que les scènes sont plutôt réalistes et que les pratiques sexuelles de la série pourraient totalement arriver dans la vie réelle ».

18. Sabine Chalvon-Demarsay, «Enquête sur des publics particulièrement concernés. La réception de L'instit et d'Urgences ", in Les Sens du public : publics politiques, publics médiatiques, éd. Daniel Céfaï et Dominique Pasquier, Paris, PUF-CURAPP, 2003, p. 501-521. L'enthousiasme unanime exprimé par les classes d'élèves interrogés par Sabine Chalvon-Demarsay sur la proximité de la série L'instit avec l'univers «dans lequel ils vivent quotidiennement» (p.507) laisse ainsi progressivement place à l'expression de « doutes » sur le « réalisme » de la série, d' indices » de son « invraisemblance » et de sa légèreté d'un point de vue éthique (p. 508).

19. Ibid., p. 519.

20. «Ce qui permet aux spectateurs peu à l'aise devant ce genre de scènes [de sexe] de se sentir à l'aise et de rire » [anonyme].

21. Gilles Deleuze, Pourparlers. 1972-1990, Paris, Minuit, 1990, p. 137.

22. Edgar Morin, Le cinéma ou l'homme imaginaire, Paris, Minuit, 1952, p. 112 : « Cette participation polymorphe que le cinéma apporte incomparablement embrasse et unit donc non seulement un personnage, mais les personnages, mais les objets, mais les paysages, mais l'univers du film dans son ensemble. Nous participons, par-delà les aventures des héros, à une totalité d'êtres, choses, actions que charrie le film dans son flux.». Ce caractère polymorphe de l'identification est presque systématiquement oublié par certaines études de réception, y compris parfois par Morin lui-même : Les stars, Paris, Seuil, 1957.

23. Ce que relève ironiquement une des contributrices en commentant l'épisode qui met en scène le coït des parents d'Hannah sous la douche, « Malgré tout, ce n'est pas le genre de scènes (série) que je regarderais volontiers avec mes parents :-)».

24. «Je ne me suis jamais posé la question ».

25. Sur les 37 étudiants interrogés ( 14 femmes, 23 hommes), et selon les 33 réponses obtenues, la moitié vient de la classe ouvrière, l'autre de la classe moyenne, voire supérieure pour une extrême minorité.

26. Sandra Laugier, «Présentation. L'autre voie de la philosophie morale ", in La voix et la vertu. Variétés du perfectionnisme moral, éd. Sandra Laugier, Paris, PUF, coll. «Éthique et philosophie morale », 2010, p. 1-30.

27. La consigne de la séance 5 était de parler des personnages les plus et les moins appréciés en raison de leurs conduites, actions et comportements.

28. Pasquier, «"Chère Hélène". Les usages sociaux des séries collège ", art. cit., p. 35.

29. Erving Goffman, Frame Analysis. An Essay on the organization of Experience, New York, Harper and Row, 1974, p. 269. Il distingue la «person-role formula » et la « role character formula », la première définissant la convenance perçue entre le rôle et la personne, la seconde la relation que nous établissons entre l'individu et son "personnage ", les rôles qu'il embrasse dans la fiction et dans la vie sociale. Sur ce point complexe, voir Laurent Jullier et Jean-Marc Leveratto, «Delon and Performance: Emploi and the Interaction between Individual, Role and Character ", in Alain Delon. Style, Stardom and Masculinity, eds. Nick Rees-Roberts and Darren Wilson, New York, Bloomsbury Academic, 2015, p. 31-42. 
30. À la question « Comment choisissez-vous les séries que vous regardez, quels sont vos critères de sélection? », Alice répond «Si un ou une actrice que j'apprécie particulièrement joue dans la série $[. .]$.$» .$

\section{RÉSUMÉS}

La série télévisée américaine Girls (HBO, 2012-17), produite par Judd Apatow, écrite par Lena Dunham (qui en est également l'actrice principale, la réalisatrice et la co-productrice) met en scène «l'entrée dans la vie » de quatre jeunes femmes installées à New York et leur recherche, tout à la fois, d'un emploi et de leur identité sexuelle. La valorisation de l'expérience féminine, comprise en tant que vécu et en tant qu'agency, particularise la série. Elle se prête particulièrement bien à une étude, dans l'esprit de Stanley Cavell, de ce que nous pouvons apprendre de l'expérience que nous faisons d'un film.

Les échanges écrits et oraux organisés dans le cadre d'un cours universitaire servent de dispositif d'observation de l'appropriation, par des étudiants français (21-26 ans), des situations mises en scène dans la première saison de la série.

Girls (HBO, 2012-17), produced by Judd Apatow, written by Lena Dunham (who was also director, coproducer, and leading actress) deals with the "Coming of age" of four young women living in New York, looking for a job and for sexual accomplishment. The series promotes their personal experience, both in the sense of lived experience and agency. It fits perfectly with Stanley Cavell's reflexion on how we can learn from our experience of viewing a movie.

This article is based on the organization, within an academic course, of a conversation with and between students (aged 21-26), as a means to observe the personal appropriation by young French adults of the situations of ordinary life staged in the first season of this series.

\section{INDEX}

Keywords : expertise, moral, ordinary, reception, sexuality

Mots-clés : expertise, morale, ordinaire, réception, sexualité

\section{AUTEURS}

\section{ELODIE VALKAUSKAS}

Elodie Valkauskas est doctorante en histoire du cinéma, elle travaille sur « Le "nouveau cinéma allemand" en France : histoire d'un label de qualité (1962-1984) » sous la direction de Fabrice Montebello, Université de Lorraine, Laboratoire Lorrain de Sciences Sociales (2L2S). Elle a été attachée temporaire d'enseignement et de recherche au département arts de Metz. Principales publications : « La diffusion des films allemands en France dans les années 1960 » (Le Portique, $\left.n^{\circ} 41,2018\right)$, « Les coproductions ouest-allemandes sur le marché cinématographique français de 1962 à 1969 » (in Les coproductions intraeuropéennes depuis 1945, eds. Valérie Pozner and Paola Palma, AFRHC, 2019) et « Jean-Marie Straub et la Moselle : histoire et réception » (in Paysage(s) de 
l'étrange. Arts et recherche sur les traces de l'histoire du Grand Est, eds. Susanne Müller and Aurélie Michel, Le Bord de l'eau, 2018).

Elodie Valkauskas is a PhD student working on "The New German Cinema in France: history of a quality label (1962-1984)" under the supervision of Pr. Fabrice Montebello at the University of Lorraine, Metz, France, in the Lorrain's Laboratory of Social Sciences (2L2S). She was Temporary Lecturer and Research Assistant and she teaches in the University of Lorraine Arts Department in Metz. Main publications: «La diffusion des films allemands en France dans les années 1960 » (Le Portique, $n^{\circ} 41,2018$ ), « Les coproductions ouest-allemandes sur le marché cinématographique français de 1962 à 1969 » (in Les coproductions intraeuropéennes depuis 1945, eds. Valérie Pozner and Paola Palma, AFRHC, 2019) and « Jean-Marie Straub et la Moselle : histoire et réception » (in Paysage(s) de l'étrange. Arts et recherche sur les traces de l'histoire du Grand Est, eds. Susanne Müller and Aurélie Michel, Le Bord de l'eau, 2018).

\section{JEAN-MARC LEVERATTO}

Jean-Marc Leveratto est professeur émérite de sociologie à l'Université de Lorraine. Ses objets de recherche sont les techniques du corps, l'histoire et la sociologie de l'industrie du spectacle (théâtre et cinéma) et la sociologie de l'expertise culturelle. Principales publications : La mesure de l'art. Sociologie de la qualité artistique (La Dispute, 2000), Introduction à l'anthropologie du spectacle, (La Dispute, 2006), Internet et la sociabilité littéraire (avec Mary Leontsini, Éditions de la BPI, 2008), Cinéphiles et cinéphilies (avec Laurent Jullier, Armand Colin, 2010). Il est membre du Laboratoire Lorrain de Sciences Sociales (2L2S).

Jean-Marc Leveratto is Emeritus professor of sociology at the University of Lorraine. His research fields are the body techniques, the history and sociology of cultural industries (theatre and cinema) and the sociology of expertise in the cultural field. He has realized numerous surveys on cultural practices (from amateur arts to cultural uses of the Internet). Main publications: $L a$ mesure de l'art. Sociologie de la qualité artistique (La Dispute, 2000), Introduction à l'anthropologie du spectacle, (La Dispute, 2006), Internet et la sociabilité littéraire (with Mary Leontsini, Éditions de la BPI, 2008), Cinéphiles et cinéphilies (with Laurent Jullier, Armand Colin, 2010). He is a member of the Laboratoire Lorrain de Sciences Sociales (2L2S). 